\title{
Kinetic analysis of protein aggregation monitored by real-time 2D solid-state NMR spectroscopy
}

\author{
Manuel Etzkorn • Anja Böckmann • \\ Marc Baldus
}

Received: 20 October 2010/Accepted: 1 January 2011/Published online: 21 January 2011

(c) The Author(s) 2011. This article is published with open access at Springerlink.com

\begin{abstract}
It is shown that real-time 2D solid-state NMR can be used to obtain kinetic and structural information about the process of protein aggregation. In addition to the incorporation of kinetic information involving intermediate states, this approach can offer atom-specific resolution for all detectable species. The analysis was carried out using experimental data obtained during aggregation of the 10.4 kDa Crh protein, which has been shown to involve a partially unfolded intermediate state prior to aggregation. Based on a single real-time $2 \mathrm{D}{ }^{13} \mathrm{C}-{ }^{13} \mathrm{C}$ transition spectrum, kinetic information about the refolding and aggregation step could be extracted. In addition, structural rearrangements associated with refolding are estimated and several different aggregation scenarios were compared to the experimental data.
\end{abstract}

M. Etzkorn · M. Baldus

Department of NMR-Based Structural Biology,

Max-Planck-Institute for Biophysical Chemistry,

Am Fassberg 11, 37077 Göttingen, Germany

\begin{abstract}
A. Böckmann
Institut de Biologie et Chimie des Protéines, UMR 5086 CNRS-UCBL, Université de Lyon,

7, passage du Vercors, 69367 Lyon, France
\end{abstract}

\section{Baldus ( $\square)$}

Bijvoet Center for Biomolecular Research, Utrecht University, Padualaan 8, 3584 Utrecht, The Netherlands

e-mail:m.baldus@uu.nl

Present Address:

M. Etzkorn

Department of Biological Chemistry and Molecular

Pharmacology, Harvard Medical School, 240 Longwood Ave,

Boston, MA 02115, USA
Keywords Aggregation - Kinetic · Solid-state NMR · Real-time spectroscopy $\cdot$ Crh

\section{Introduction}

Protein aggregation has become recognized as an important aspect of the protein folding landscape. This process not only interferes with protein expression and recovery assays used in biotechnology, but also impacts the every day live of cells and organisms and is associated with a variety of human diseases (Dobson 2003; Wetzel 2006). Methods to characterize aggregation kinetics comprise predominantly biochemical and biophysical approaches such as gel filtration, sedimentation assays, binding of fluorescent markers, AFM imaging, dynamic light scattering or circular dichroism that report on the oligomerisation state or the overall secondary structure content of the protein (Hurshman et al. 2004; Wetzel 2006).

Real-time solution-state NMR has shown to be useful to follow protein and RNA folding at the level of individual residues (Balbach et al. 1996; Van Nuland et al. 1998; Zeeb and Balbach 2004; Corazza et al. 2010; Lee et al. 2010). In principle, solid-state NMR (ssNMR) offers a complementary spectroscopic means to probe structural and kinetic aspects of protein folding and aggregation, especially as molecular aggregates increase in size. Indeed, ssNMR, has made great progress to structurally study trapped intermediate states of Amyloid (Chimon et al. 2007; Ahmed et al. 2010) and globular (Hu and Tycko 2010) proteins or to examine the effect of protein mutations known to interfere with protein aggregation (Heise et al. 2008; Karpinar et al. 2009; Kim et al. 2009). In principle, kinetic information becomes accessible by repeating ssNMR on cryotrapped intermediates at different time points or by recording NMR 
data directly during refolding. Indeed, time-resolved 1D ssNMR has been used to detect signal intensity buildup or decay during protein aggregation (Kamihira et al. 2000) and ATP hydrolysis of an ABC transporter (Hellmich et al. 2008) or by combining ssNMR pulse schemes with diffusion measurements using PFGs (Ader et al. 2010). Due to limited spectral resolution 1D ssNMR has to be combined with specific labeling techniques to offer site-specific resolution in proteins. Earlier, we have shown (Etzkorn et al. 2007) that for the Crh protein from B. subtilis, molecular aggregation triggered by a small temperature jump can be followed by two-dimensional ssNMR. Starting from a kinetically destabilized protein precipitate, protein aggregation led to significant increase in $\beta$-sheet content, whereas smaller $\alpha$-helical fragments were retained in the aggregated state. Using Crh as an example, we here demonstrate that 2D ssNMR data sets recording these structural rearrangements in real time offer structural and, in particular, kinetic information about the process of protein aggregation. In general our analysis can offer atom-specific resolution for large segments of the protein and can simultaneously detect and kinetically describe a range of possible intermediate states during protein aggregation under conditions where molecular size or density prohibit the application of other biophysical methods.

\section{Theory, materials and methods}

Real-time NMR spectroscopy is sensitive to modulations of the signal induced by a transition between two or more conformational states present in the sample during acquisition. In general these modulations derive from changes in the population profile during the transition. This kinetic profile directly affects the time evolution for each spin and the resulting spectrum can be written (separately for a single spin in different states) as:

$\operatorname{Re}(S(\omega))=\int_{0}^{\infty}\left(\sum_{n} a_{n} P_{n}\left(k_{i}, t\right) \cdot e^{\left(2 \pi i \omega_{n}-\lambda_{n}\right) t}\right) \cdot e^{-2 \pi i \omega t} d t$

While the scaling factors $a_{n}$ account for variations in signal intensity among the different states $n$, the parameters $\lambda_{\mathrm{n}}$ are determined by the transverse spin relaxation rates. The kinetic profile $P_{n}\left(k_{j}, t\right)$ reports on the population of state $n$ characterized by resonance frequency $\omega_{n}$ at a given time and depends on one or several rate constants $k_{j}$ describing the transition.

Normally, time frame, resolution and sensitivity offered by a single 1D NMR experiment are insufficient for the investigation of protein aggregation. A series of 1D spectra with sufficient repetition steps recorded during the transition (time-resolved NMR spectroscopy) can increase sensitivity and might offer an appropriate observation window. However, site specific resolution for larger fractions of the protein or even the detection of an intermediate species can be difficult to achieve. Instead, multidimensional spectroscopy can be used to follow the transition during the evolution of an indirect dimension (real-time NMR spectroscopy). Under the assumption that the transition during the repetitions of a single time step is negligible small, the spectrum after Fourier transformation (FT) in the direct dimension remains unaffected, whereas the peak shape and amplitude in the indirect dimension is modified. For a 2D spectrum (1) reads as:

$$
\begin{aligned}
& \operatorname{Re}\left(S\left(\omega_{1}, \omega_{2}\right)\right) \\
& =\int_{0}^{\infty}\left(\sum_{n} a_{n} P_{n}\left(k_{i}, t\right) \cdot e^{\left(2 \pi i \omega_{1, n}-\lambda_{1, n}\right) t} \cdot L_{n}\left(\omega_{2, n}\right)\right) \cdot e^{-2 \pi i \omega t} d t
\end{aligned}
$$

Note that (2) already includes the FT in the direct dimension $\left(t_{2}\right)$ leading to the Lorentzian functions $L_{n}\left(\omega_{2, n}\right)$ for the states $n$. An exponential decay of state A during the evolution period in the indirect dimension is mathematically equivalent to an increased line-width for state $\mathrm{A}$, whereas an exponential increase in the population leads to a baseline distortion and the occurrence of negative 'shoulders' of the corresponding peaks. These characteristic features are typically found experimentally in real-time NMR during protein folding in solution (Balbach et al. 1996).

For analysis relevant $1 \mathrm{D}$ extracts of real-time 2D spectra are usually fitted to theoretically simulated peak shapes (Balbach et al. 1996; Helgstrand et al. 2000) or a direct analytical solution (Balbach et al. 1999; Zeeb and Balbach 2004). Due to increased spectral overlap in ssNMR, we used (2) to develop a Mathematica (Wolfram scientific) script to calculate the difference of a full theoretical 2D cross peak pattern and the experimental data. Different transition scenarios were examined by varying the mathematical description of the kinetic profile. Amplitude factors $a_{n}$ were set to one, implying the same transfer efficiency for all states. Free theoretical parameters such as $\lambda_{(1 ; 2), \mathrm{n}}$ and $\omega_{(1 ; 2), \mathrm{n}}$ were chosen according to the line width and position of the peak maxima in the experimental spectrum.

\section{Simulating real-time 2D spectra}

Mathematica (Wolfram scientific) version 6.0.1 was used to fit the experimental data to simulated peak patterns by numerically integrating (FT) $S\left(t_{1}, \omega_{2}\right)$ (2) for the population profiles considered. 'SymbolicProcessing' was switched off to speed up the integration process. The experimental spectrum was processed using exponential line broadening of $50 \mathrm{~Hz}$. The underlying window function 
was also implemented in the simulations before FT in the indirect dimension. The effect in the direct dimension was neglected. Resonance frequencies of the occurring states were taken from corresponding cross signals in the experimental spectrum. The line width was measured using the Thr $\mathrm{C} \beta-\mathrm{C} \gamma 2$ cross peaks, which in the real-time ${ }^{13} \mathrm{C}-{ }^{13} \mathrm{C} 2 \mathrm{D}$ spin diffusion spectrum are not symmetric to the Thr $\mathrm{C} \gamma 2-\mathrm{C} \beta$ peaks. Here the line width in the direct dimension is resolved and should be largely unaffected by the transition. The line width was comparable for all states, hence justifying in part the assumption $a_{n}=1$ for all $n$.

\section{Calculating difference plots}

Experimental data were taken from Etzkorn et al. (2007). Signal intensity in the simulated spectra was read out at the corresponding data points obtained by processing the whole experimental spectrum with 512 points in $\omega_{1}$ and 2,048 in $\omega_{2}$. The spectral extract of the $\mathrm{Thr} \mathrm{C} \gamma 2-\mathrm{C} \beta$ $(\mathrm{C} \beta-\mathrm{C} \gamma 2)$ cross section consists of $31 \times 21(21 \times 31)$ values which were treated individually. Free parameters of the kinetic profile were varied in nested loops.

\section{Conformational analysis}

To evaluate the subset of the torsion angle space that is in agreement with the experimentally observed $\mathrm{Thr} \mathrm{C} \beta$ shift we generated a set of heptapeptides (AATAA) by varying the Thr $\psi$ and $\phi$ torsion angle in steps of $10^{\circ}$. ShiftX (Neal et al. 2003) was used to predict the expected chemical shift for each peptide. Chemical shift intervals [68.4, $70 \mathrm{ppm}$ ], [69.7, $70.8 \mathrm{ppm}]$ and $[70.4,72.5 \mathrm{ppm}]$ were chosen to select a match for the states A, B and C, respectively.

\section{Results}

As described in Etzkorn et al. (2007), protein aggregation of the Crh protein induced by a modest temperature increase could be detected in real time during a $2 \mathrm{D}\left({ }^{13} \mathrm{C}\right.$, ${ }^{13} \mathrm{C}$ ) ssNMR experiment. The transition can be readily followed using cross-signal intensities in the $\mathrm{Thr} \mathrm{C} \gamma 2-\mathrm{C} \beta$ region. As visible in Fig. 1 the transition spectrum reveals resolved peaks for the initial state A (natively folded), an intermediate state B (partially unfolded) and a final state C (aggregated). Hence, application of (2) involves summation over three states $(n=A, B, C)$.

\section{Conformational analysis}

As shown before (Havlin and Tycko 2005; Heise et al. 2005) cross peak positions and line width offer a spectroscopic means to estimate the changes in backbone structure

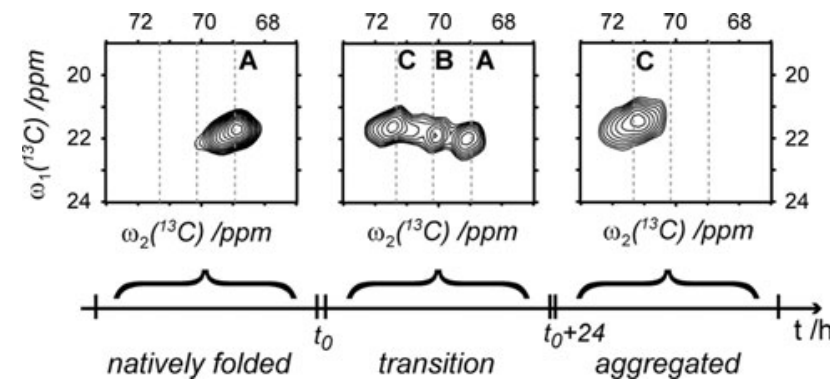

Fig. 1 Experimental ssNMR data (as reported in ref Etzkorn et al. (2007) of the Thr $\mathrm{C} \gamma 2-\mathrm{C} \beta$ region of $\mathrm{Crh}$ recorded before the transition (left), during the transition (middle) and afterwards (right). Refolding was induced by increasing the temperature by $13^{\circ} \mathrm{C}$ at time $t_{0}$. The time delay between spectrum 1 (native) and 2 (transition) was about $15 \mathrm{~min}$ to allow for system equilibration and readjustment of spectroscopic parameters. Spectra 2 and 3 were recorded without any additional time delay. In each case, data were acquired for about $24 \mathrm{~h}$

resulting from protein refolding. In the case of the Thr $\mathrm{C} \gamma 2-\mathrm{C} \beta$ region, a torsion angle analysis for the involved states is presented in Fig. 2. In Fig. 2a, a subset of torsion angles which agree, according to shiftX (Neal et al. 2003), with the measured Thr $\mathrm{C} \beta$ peak position is shown. Note that dihedral angles predicted for the A-state (blue circles) agree well with values predicted from the X-ray structure for involved Threonines (black crosses, see also Etzkorn et al. 2004). An additional refinement of torsion angle space sampled during refolding can be obtained by taking into account allowed regions of the Ramachandran plot (Fig. 2b) and by considering only lowest energies assuming an amino-acid specific torsion angle potential as given in Kuszewski and Clore (2000) (Fig. 2c). Especially for the latter case, we find that the observed changes in the $2 \mathrm{D}$ ssNMR spectra can be explained by rather small changes in (Phi, Psi) space, at least in the case of Thr resonances.

Crh in a classical three-state folding transition

For the Crh protein, three separate states could be detected in the real-time spectrum. Since the detected folding process is not reversible, we did not consider chemical exchange as a dominant effect to explain our experimental results. Instead, we applied a classical protein folding scenario (Balbach et al. 1996) in which the two underlying transitions are expected to be single exponential, leading to a kinetic profile of the form:

$P_{A}\left(k_{1}, t\right)=e^{-k_{1} t}$
$P_{B}\left(k_{1}, k_{2}, t\right)=\left(1-e^{-k_{1} t}\right) e^{-k_{2} t}$
$P_{C}\left(k_{1}, k_{2}, t\right)=\left(1-e^{-k_{1} t}\right)\left(1-e^{-k_{2} t}\right)$

Figure 3a shows the resulting difference plot as a function of the two rate constants $k_{1}$ for unfolding and $k_{2}$ for 

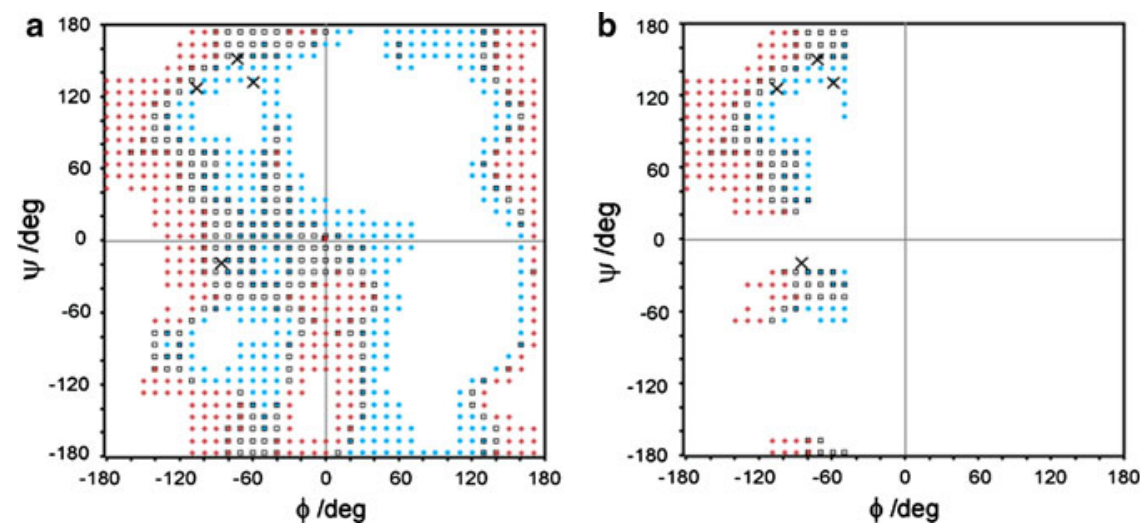

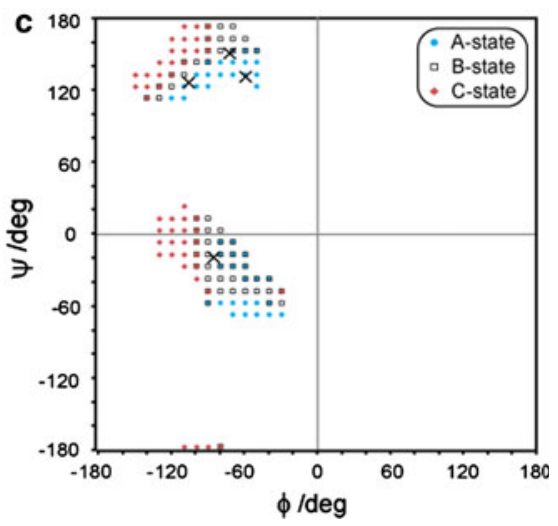

Fig. 2 Torsion angle analysis for the involved states. a Subset of torsion angles which agree, according to shiftX (Neal et al. 2003), with the measured Thr $\mathrm{C} \beta$ peak position (blue circles A-state; empty rectangles $\mathrm{B}$-state; red diamonds $\mathrm{C}$-state; black crosses as found in the X-ray structure for involved Threonines). b Torsion angles that are consistent with the peak position and fall in allowed regions of the classical Ramachandran plot. $\mathbf{c}$ Torsion angles that are consistent with the peak position and have most favorable energies assuming an amino-acid specific torsion angle potential as given in Kuszewski and Clore (2000) aggregation. The resulting kinetic profile and spectral comparison of the best fit are given in Fig. 3d, g, respectively. The timescale of the simulated data and hence the absolute values for the involved rate constants have to be corrected for the difference of the indirect acquisition time and the time of the experiment, according to:

$k_{\mathrm{eff}}=\frac{t_{\mathrm{NMR}}}{t_{\mathrm{real}}} \cdot k_{\mathrm{NMR}} \approx 8.1 \times 10^{-8} \cdot k_{\mathrm{NMR}}$

The global minimum in Fig. $3 \mathrm{a}$ is given for the effective rate constants $k_{1}=1.36 \times 10^{-4} \mathrm{~s}^{-1}$ and $k_{2}=0.58 \times$ $10^{-4} \mathrm{~s}^{-1}$. Since the best fit (Fig. $3 \mathrm{~g}$ ) still shows some considerable differences to the experimental data, calculation of the error margin is not attempted here. However, the transition predominantly interferes with the acquisition of the indirect dimension rendering the $\left({ }^{13} \mathrm{C},{ }^{13} \mathrm{C}\right)$ spectrum asymmetric and cross peaks on different sides of the diagonal offer an independent set of information.

Figure $3 \mathrm{~b}$ shows the difference plot for the Thr $C \beta-C \gamma 2$ cross correlations. The minimum is found for $k_{1}=0.77 \times$ $10^{-4} \mathrm{~s}^{-1}$ and $k_{2}=0.52 \times 10^{-4} \mathrm{~s}^{-1}$. Combination of data from both sides of the diagonal leads to the difference plot shown in Fig. 3c. The respective population profiles according to the best fit rate constants allow for an estimation of the accuracy of the method. The rate constants obtained from Fig. $3 \mathrm{c}$ are $k_{1}=0.91 \times 10^{-4} \mathrm{~s}^{-1}$ and $k_{2}=0.65 \times 10^{-4} \mathrm{~s}^{-1}$. Notably, the comparison of the theoretical spectra to the experimental ones (Fig. $3 \mathrm{~g}, \mathrm{~h}$ ) shows that characteristic features such as line broadening for the initial as well as baseline distortions for the final state are significantly less reproduced by the experimental data than expected for the considered single exponential transition.
Crh in a classical aggregation scenario

The single exponential transition used to describe protein folding (Balbach et al. 1996) is in general not sufficient to kinetically describe an aggregation process since it does not account for a nucleation step. Instead, several mechanisms and their mathematical formalisms have been suggested to describe experimentally determined data of protein aggregation (see, e.g. Thusius et al. 1975; Wegner and Engel 1975; Frieden and Goddette 1983; Eigen 1996; Ferrone 1999; Morris et al. 2008). In general, measurement of a population profile is not sufficient to reveal the underlying mechanism (Ferrone 1999). In the following the "Finke-Watzky" (F-W) model of protein aggregation (Watzky and Finke 1997) was selected to theoretically describe the kinetic profile. The F-W model assumes a 2-step mechanism involving a (slow) continuous nucleation with rate constant $k_{a 1}$, followed by a (fast) autocatalytic surface growth with the rate constant $k_{a 2}$. It was shown that this minimalistic kinetic model is able to account for a broad range of protein aggregation (Morris et al. 2008; Morris et al. 2009). The most convenient form for analyzing the experimental data was suggested (Morris et al. 2008) to be:

$$
[C]=[B]_{0}-\frac{\frac{k_{a 1}}{k a_{2}}+[B]_{0}}{1+\frac{k_{a 1}}{k_{a 2}[B]_{0}} e^{\left(k_{a 1}+k_{a 2}[B]_{0}\right) t}}
$$

where (5) only describes the aggregation process from the (unfolded) intermediate to the final aggregated state $(\mathrm{B} \rightarrow \mathrm{C})$. The following time dependence of the relative populations was hence used to fit the experimental data: 

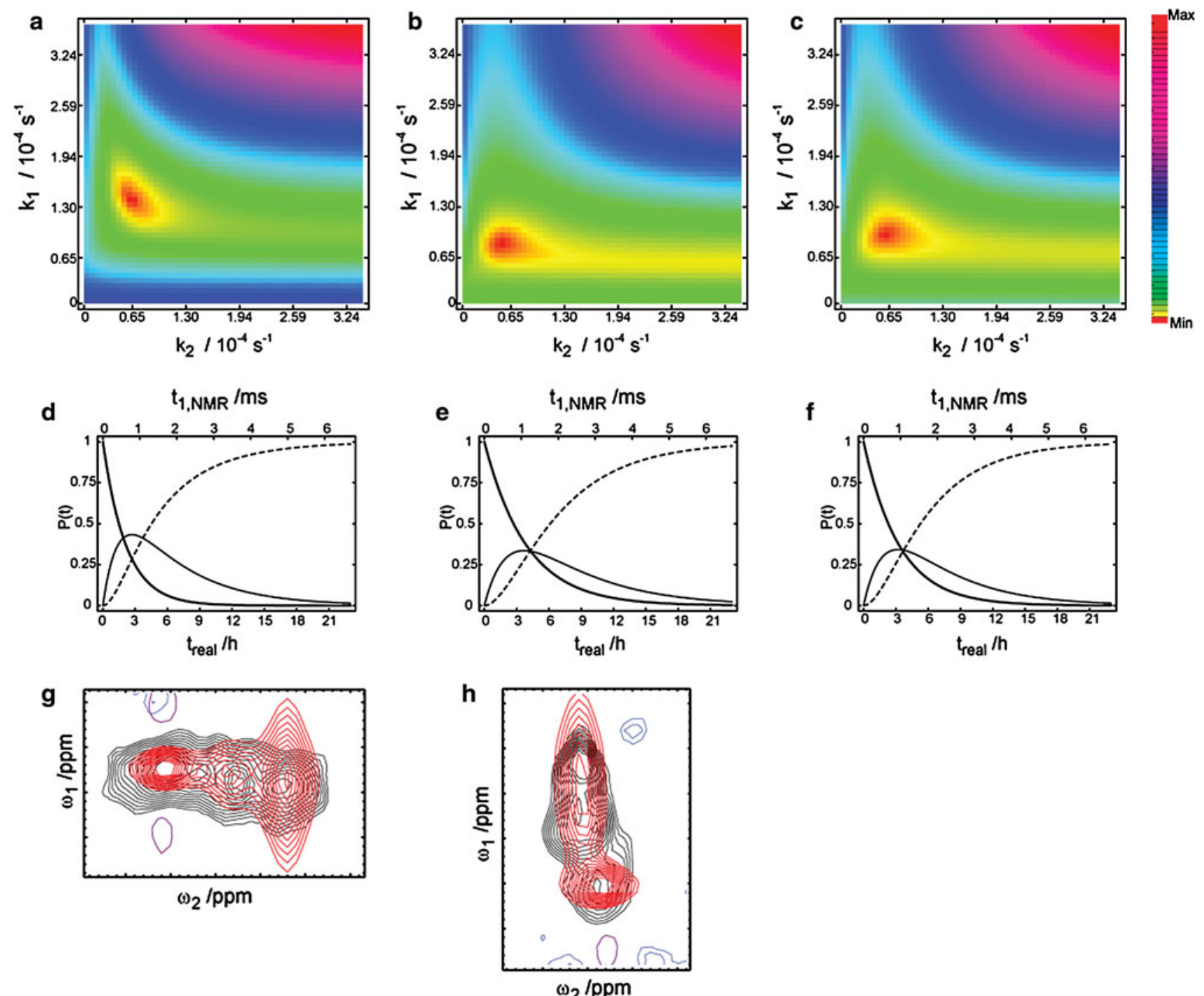

Fig. 3 Results of the kinetic analysis assuming single exponential transitions. a-c Difference plot between normalized experimental data and theoretically calculated $\mathrm{Thr} \mathrm{C} \gamma 2-\mathrm{C} \beta$ cross signals as a function of both rate constants. For each pair of $k_{1}$ and $k_{2}$, the sum of the absolute value of the difference over all considered points in the 2D spectrum is plotted (see "Theory, materials and methods" for

$$
P_{A}(t)=e^{-k_{1} t}
$$

$P_{B}\left(k_{a 1}, k_{a 2}, t\right)=\left(1-e^{-k_{1} t}\right) \frac{\frac{k_{a 1}}{k_{k_{2}}}+1}{1+\frac{k_{a 1}}{k_{a 2}} e^{\left(k_{a 1}+k_{a 2}\right) t}}$

$P_{C}\left(k_{a 1}, k_{a 2}, t\right)=\left(1-e^{-k_{1} t}\right)\left(1-\frac{\frac{k_{a 1}}{k_{a 2}}+1}{1+\frac{k_{a 1}}{k_{a 2}} e^{\left(k_{a 1}+k_{a 2}\right) t}}\right)$

Figure 4 summarizes the results obtained with this model. The three-dimensional contour maps shown in Fig. 4a-c, visualize areas which are within 5\% (red), 10\% (pale yellow) and $15 \%$ (white) deviation from the minimum more details). Population profile (d-f) and comparison of spectral extract of the resulting best fit $(\mathbf{g}, \mathbf{h})$. Positive/negative contours of the experimental spectrum are given in black/blue, whereas theoretical data are given in red/purple. b As in a but for $\mathrm{Thr} \mathrm{C} \beta-\mathrm{C} \gamma 2$ cross signal. c Normalized sum of $\mathbf{a}$ and $\mathbf{b}$

difference between experimental and simulated cross section of Thr $\mathrm{C} \gamma 2-\mathrm{C} \beta$ (a), Thr $\mathrm{C} \beta-\mathrm{C} \gamma 2$ (b) and both (c). The resulting population profile of the minimum is shown in Fig. $4 \mathrm{~d}-\mathrm{f}$, respectively. Figure $4 \mathrm{~g}$, h compare the best fit (according to $\mathrm{c}$ and $\mathrm{f}$ ) to the experimental data.

Notably, a comparison to the best fit according to a single exponential three-state transition (Fig. 3a, b, lower part), reveals that the assumption of a nucleation step here did not improve the fitting. Indeed the line broadening of the initial state is predominantly related to the unfolding mechanism and, as evident from Fig. 4g, h, not reproduced even by the best fit of the profiles represented by (6a-6c). 

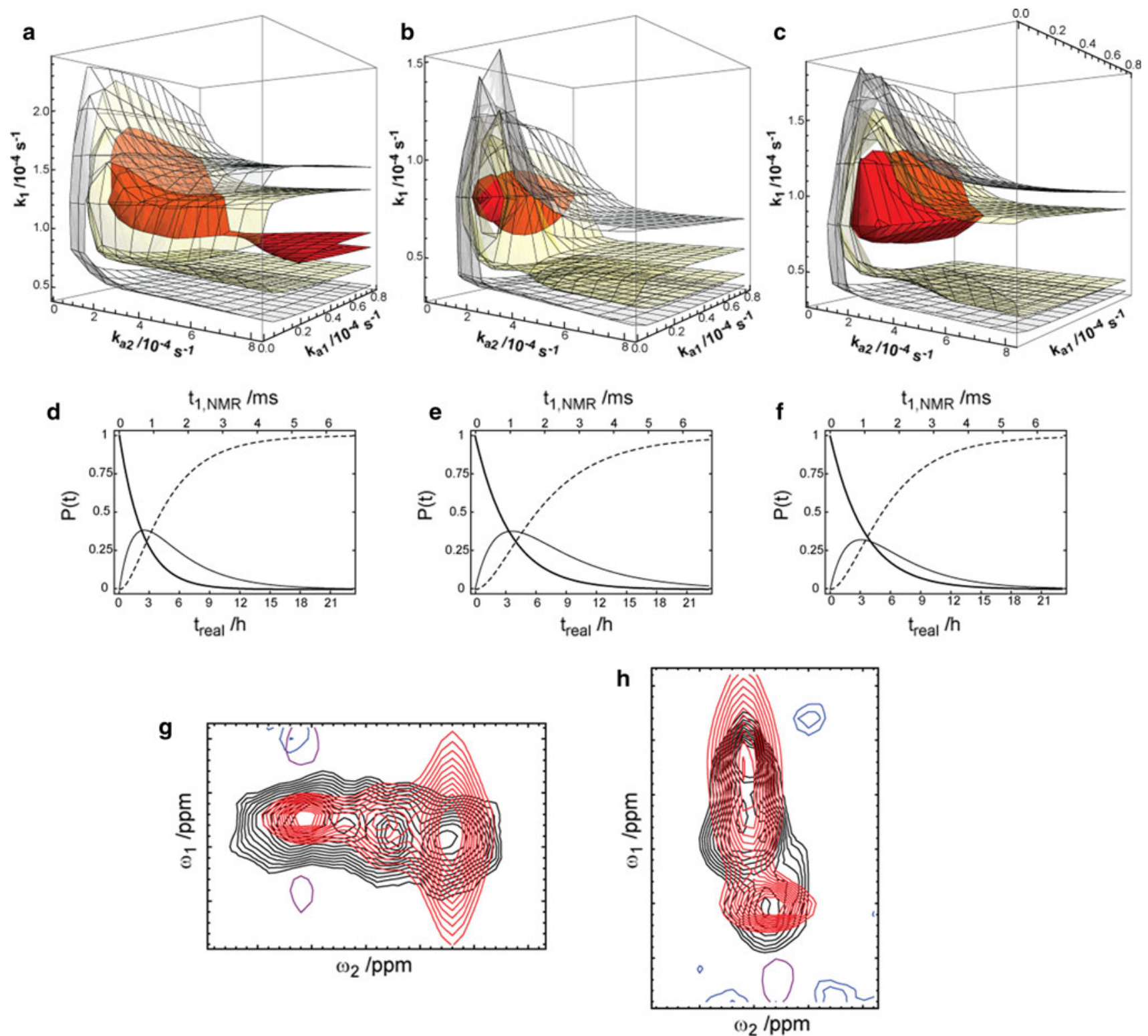

Fig. 4 Results of the kinetic analysis using the F-W model. a-c Three-dimensional contour maps of the difference between experimental and simulated data depending on the three parameters $k_{1}, k_{a 1}$ and $k_{a 2}$. Red, yellow and white contour levels indicate areas within 5, 10 and $15 \%$ deviation to the best fit. The results for the Thr

Crh in a stretched exponential unfolding scenario

It has been reported that under specific circumstances a single exponential function is not sufficient to describe protein (un)folding (Sabelko et al. 1999). Phenomenological, these findings were related to a small or absent energy barrier separating the initial and final state (Garcia-Mira et al. 2002; Osváth et al. 2003; Nakamura et al. 2004). The absence of a distinct energy barrier can lead to a variety of possible transitions, each reflected by a different rate constant. Multiple or stretched exponential functions can
$\mathbf{C} \gamma 2-\mathrm{C} \beta / \mathrm{Thr} \mathbf{C} \beta-\mathrm{C} \gamma 2$ cross section are shown in $\mathbf{a} / \mathbf{b}$, whereas $\mathbf{c}$ shows the sum of both. The kinetic profiles of the best fit are given in $\mathbf{d}-\mathbf{f}$, respectively. g, h Compare the experimental data to the best fit according to $\mathbf{c}$ and $\mathbf{f}$. Color code as in Fig. $3 \mathbf{g}, \mathbf{h}$

be used to account for the occurring effects (Nakamura et al. 2004; Ma and Gruebele 2005). Introducing only one additional parameter $b \varepsilon[0,1]$ a stretched exponential function was used to describe the protein unfolding as follows:

$P_{A}\left(k_{1}, b, t\right)=e^{-\left(k_{1} t\right)^{b}}$

$P_{B}\left(k_{a 1}, k_{a 2}, b, t\right)=\left(1-e^{-\left(k_{1} t\right)^{b}}\right) \frac{\frac{k_{a 1}}{k_{a 2}}+1}{1+\frac{k_{a 1}}{k_{a 2}} e^{\left(k_{a 1}+k_{a 2}\right) t}}$ 

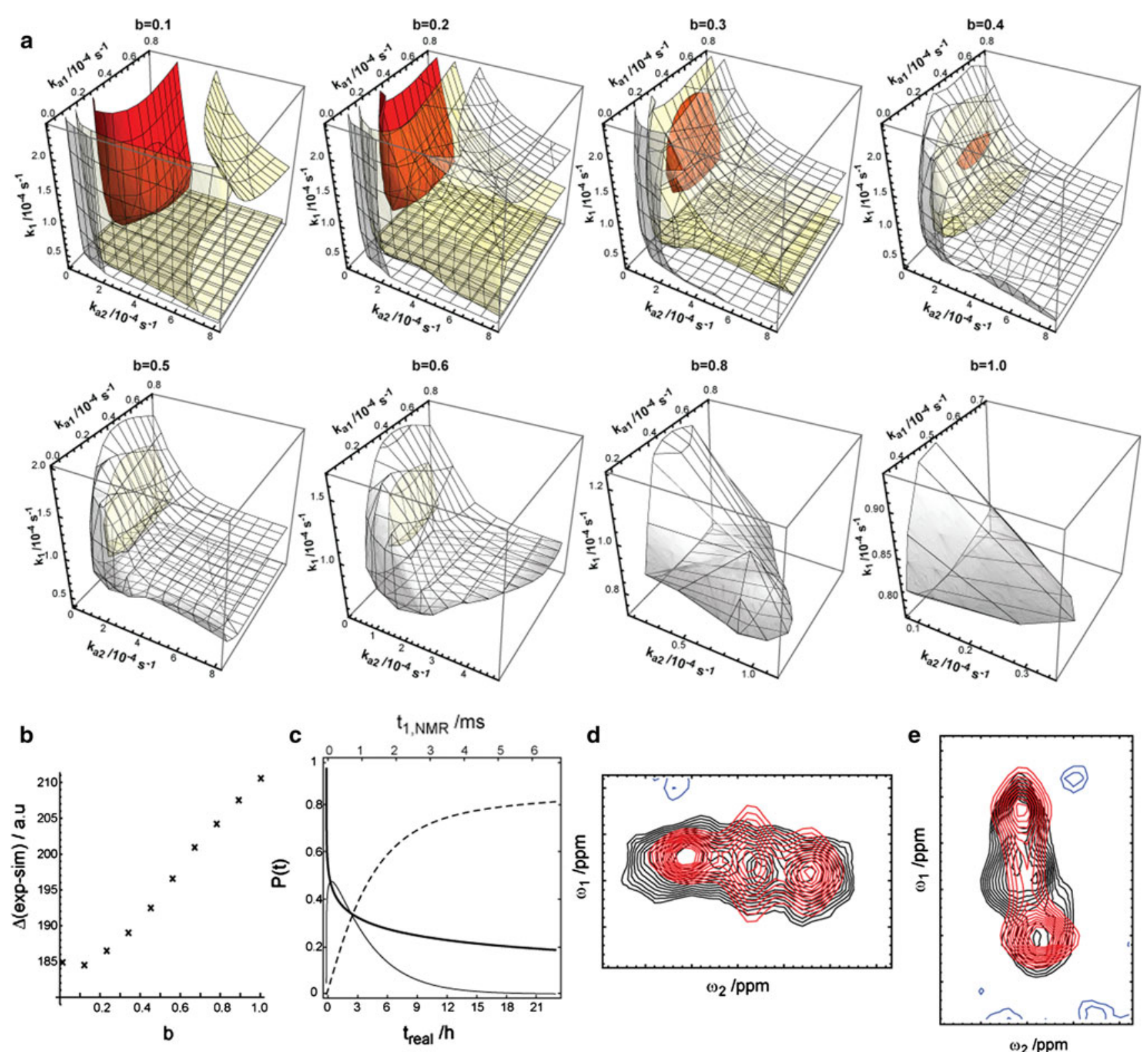

d
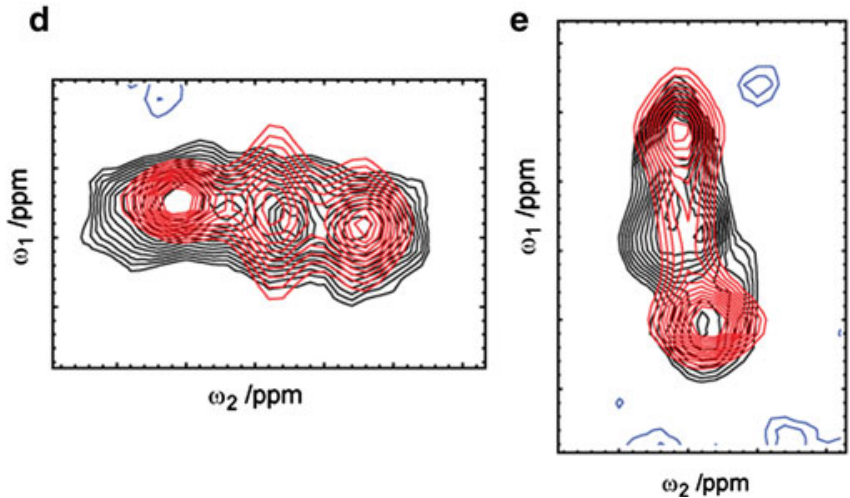

Fig. 5 Kinetic analysis assuming downhill unfolding and the F-W model for aggregation. a 3D contour maps as given in Fig. 4c, but for indicated values of $b$. The minimal difference found to the

$P_{C}\left(k_{a 1}, k_{a 2}, b, t\right)=\left(1-e^{-\left(k_{1} t\right)^{b}}\right)\left(1-\frac{\frac{k_{a 1}}{k_{a 2}}+1}{1+\frac{k_{a 1}}{k_{a 2}}\left(^{\left(k_{a 1}+k_{a 2}\right) t}\right.}\right)$

Equations $7 \mathrm{a}-7 \mathrm{c}$ contain four free parameters. The results of fitting this model to the experimental spectrum as a series of 3D contour maps depending on the parameter $b$ are shown in Fig. 5. Each 3D plot depends on the same three parameters as described in Fig. 4. Although the high number of free parameters impedes an accurate determination of the rate constants, it is clearly evident that the experimental data depending on $b$ is plotted in b. $\mathbf{c}$ The population profile of the global minimum and $(\mathbf{d}+\mathbf{e})$ the corresponding simulated peak pattern

simulated cross correlation approaches the experimental data for $b<1$. Plotting the minimal difference to the experimental data as a function of $b$ (Fig. 5b) reveals that the global minimum is found for $b=0.1$. However, also values up to $b=0.4$ may reproduce the experimental spectrum within an error of 5\%. Figure $5 \mathrm{c}-\mathrm{e}$ shows the population profile as well as the resulting peak pattern of the best fit $\left(b=0.1 ; k_{1}=2.03 \times 10^{-4} \mathrm{~s}^{-1} ; k_{a 1}=0.78 \times 10^{-4} \mathrm{~s}^{-1}\right.$; $k_{a 2}=0.81 \times 10^{-5} \mathrm{~s}^{-1}$ ). Indeed the stretched exponential unfolding scenario could explain the missing line broadening in the experimental data of state A. 
Notably, a completely heterogeneous aggregation scenario also involving a stretched exponential aggregation step as suggested for transthyretin aggregation (Hurshman et al. 2004) could also explain the data (see supporting figure SI 1). However, experimental data analyzed here exclusively report on the population profile. Additional studies on the effect of concentration of native Crh and of seeding with preaggregated Crh may help to discriminate between stretched exponential aggregation, i.e. heterogeneous growth of aggregates with the monomer as the critical nucleus size, and the F-W model, i.e. continuous nucleation followed by an autocatalytic surface growth (Ferrone 1999; Hurshman et al. 2004).

\section{Discussion}

Several methods exist that allow studying protein aggregation kinetics. Most of these techniques rely on the detection of global factors or indirect mechanisms (e.g. the $\beta$-strand content in Thioflavin $\mathrm{T}$ binding or CD spectroscopy) and may lack the formation and kinetic properties of intermediate species. Here we have shown that ssNMR can contribute to the kinetic and structural analysis of insoluble protein conformations, in particular of those including intermediate folding states which might be an important target to interfere with the aggregation process (Cohen and Kelly 2003).

While intermediate states might be rapidly frozen for a more detailed structural study (Chimon et al. 2007; Ahmed et al. 2010; Hu and Tycko 2010), real-time ssNMR offers unique possibilities to characterize aggregation kinetics. The range of observable states can be modified by a combination of different sets of polarization transfer mechanisms (e.g. based on dipolar or scalar couplings) (Andronesi et al. 2005) or by simply recording ssNMR spectra after direct excitation (Kamihira et al. 2000). Our analysis was solely based on ssNMR cross-signal intensities from a single kinetic transition to investigate the potential of the method. Changes in backbone conformation were estimated from an analysis of conformation-dependent chemical shifts. Rate constants could be extracted using a single exponential three-state transition as well as a conventional aggregation mechanism. No significant difference between the two approaches could be detected, suggesting that the formation of a nucleus is not a significant step in the aggregation of Crh protein precipitates. Remaining differences to the experimental data additionally suggest that a single exponential transition for the initial unfolding step does not suffice to properly describe the time course of the folding process. Instead, a stretched exponential function, as found in downhill folding (Sabelko et al. 1999; Nakamura et al. 2004), significantly improves the agreement between experimental and theoretical data. The stretched exponential decay would be consistent with a heterogeneous unfolding step involving one or several fast as well as slow decaying components.

While limitations regarding sensitivity and resolution do not allow for a more detailed analysis of the aggregation mechanism from ssNMR at this stage, ssNMR studies using selectively labeled protein variants and the combination with other biophysical methods will provide additional opportunities to refine kinetic and structural profiles. Such studies may not only be relevant in the context of molecular aggregation but their application may also facilitate an atomic description of inter-molecular interactions in the context of molecular gel formation (Ader et al. 2010) or protein insertion into membranes.

Acknowledgments This work was funded by NWO (Grant number 700.26.121), the CNRS (PICS no. 2424), the French research ministry (ACI Biol. Cell. Mol. Et Struct. 2003; ANR JCJC 2005; ANRPCV08_321323), and the Max Planck Gesellschaft.

Open Access This article is distributed under the terms of the Creative Commons Attribution Noncommercial License which permits any noncommercial use, distribution, and reproduction in any medium, provided the original author(s) and source are credited.

\section{References}

Ader C, Frey S, Maas W, Schmidt HB, Gorlich D, Baldus M (2010) Amyloid-like interactions within nucleoporin FG hydrogels. Proc Natl Acad Sci USA 107:6281-6285

Ahmed M, Davis J, Aucoin D, Sato T, Ahuja S, Aimoto S, Elliott JI, Van Nostrand WE, Smith SO (2010) Structural conversion of neurotoxic amyloid-beta(1-42) oligomers to fibrils. Nat Struct Mol Bio 17:556-561

Andronesi OC, Becker S, Seidel K, Heise H, Young HS, Baldus M (2005) Determination of membrane protein structure and dynamics by magic-angle-spinning solid-state NMR spectroscopy. J Am Chem Soc 127:12965-12974

Balbach J, Forge V, Lau WS, van Nuland NA, Brew K, Dobson CM (1996) Protein folding monitored at individual residues during a two-dimensional NMR experiment. Science 274:1161-1163

Balbach J, Steegborn C, Schindler T, Schmid FX (1999) A protein folding intermediate of ribonuclease $\mathrm{T} 1$ characterized at high resolution by $1 \mathrm{D}$ and $2 \mathrm{D}$ real-time NMR spectroscopy. J Mol Biol 285:829-842

Chimon S, Shaibat MA, Jones CR, Calero DC, Aizezi B, Ishii Y (2007) Evidence of fibril-like [beta]-sheet structures in a neurotoxic amyloid intermediate of Alzheimer's [beta]-amyloid. Nat Struct Mol Biol 14:1157-1164

Cohen FE, Kelly JW (2003) Therapeutic approaches to proteinmisfolding diseases. Nature 426:905-909

Corazza A, Rennella E, Schanda P, Mimmi MC, Cutuil T, Raimondi S, Giorgetti S, Fogolari F, Viglino P, Frydman L, Gal M, Bellotti V, Brutscher B, Esposito G (2010) Native-unlike long-lived intermediates along the folding pathway of the amyloidogenic protein beta(2)-microglobulin revealed by real-time two-dimensional NMR. J Biol Chem 285:5827-5835 
Dobson CM (2003) Protein folding and misfolding. Nature 426:884-890

Eigen M (1996) Prionics or the kinetic basis of prion diseases. Biophys Chem 63:A1-A18

Etzkorn M, Böckmann A, Lange A, Baldus M (2004) Probing molecular interfaces using 2D magic-angle-spinning NMR on protein mixtures with different uniform labeling. J Am Chem Soc 126:14746-14751

Etzkorn M, Böckmann A, Penin F, Riedel D, Baldus M (2007) Characterization of folding intermediates of a domain-swapped protein by solid-state NMR spectroscopy. J Am Chem Soc 129:169-175

Ferrone F (1999) Analysis of protein aggregation kinetics. Methods Enzymol 309:256-274

Frieden C, Goddette DW (1983) Polymerization of actin and actinlike systems: evaluation of the time course of polymerization in relation to the mechanism. Biochemistry 22:5836-5843

Garcia-Mira MM, Sadqi M, Fischer N, Sanchez-Ruiz JM, Muñoz V (2002) Experimental identification of downhill protein folding. Science 298:2191-2195

Havlin RH, Tycko R (2005) Probing site-specific conformational distributions in protein folding with solid-state NMR. Proc Natl Acad Sci USA 102:3284-3289

Heise H, Luca S, de Groot BL, Grubmuller H, Baldus M (2005) Probing conformational disorder in neurotensin by two-dimensional solid-state NMR and comparison to molecular dynamics simulations. Biophys J 89:2113-2120

Heise H, Celej MS, Becker S, Riedel D, Pelah A, Kumar A, Jovin TM, Baldus M (2008) Solid-state NMR reveals structural differences between fibrils of wild-type and disease-related A53T mutant [alpha]-synuclein. J Mol Biol 380:444-450

Helgstrand M, Härd T, Allard P (2000) Simulations of NMR pulse sequences during equilibrium and non-equilibrium chemical exchange. J Biomol NMR 18:49-63

Hellmich UA, Haase W, Velamakanni S, van Veen HW, Glaubitz C (2008) Caught in the act: ATP hydrolysis of an ABC-multidrug transporter followed by real-time magic angle spinning NMR. FEBS Lett 582:3557-3562

Hu K-N, Tycko R (2010) What can solid state NMR contribute to our understanding of protein folding? Biophys Chem 151:10-21

Hurshman AR, White JT, Powers ET, Kelly JW (2004) Transthyretin aggregation under partially denaturing conditions is a downhill polymerization. Biochemistry 43:7365-7381

Kamihira M, Naito A, Tuzi S, Nosaka AY, Saitô H (2000) Conformational transitions and fibrillation mechanism of human calcitonin as studied by high-resolution solid-state ${ }^{13} \mathrm{C}$ NMR. Protein Sci 9:867-877

Karpinar DP, Balija MBG, Kugler S, Opazo F, Rezaei-Ghaleh N, Wender N, Kim HY, Taschenberger G, Falkenburger BH, Heise H, Kumar A, Riedel D, Fichtner L, Voigt A, Braus GH, Giller K, Becker S, Herzig A, Baldus M, Jackle H, Eimer S, Schulz JB,
Griesinger C, Zweckstetter M (2009) Pre-fibrillar alpha-synuclein variants with impaired beta-structure increase neurotoxicity in Parkinson's disease models. EMBO J 28:3256-3268

Kim H-Y, Cho M-K, Kumar A, Maier E, Siebenhaar C, Becker S, Fernandez CO, Lashuel HA, Benz R, Lange A, Zweckstetter M (2009) Structural properties of pore-forming oligomers of alphasynuclein. J Am Chem Soc 131:17482-17489

Kuszewski J, Clore GM (2000) Sources of and solutions to problems in the refinement of protein NMR structures against torsion angle potentials of mean force. J Magn Reson 146:249-254

Lee MK, Gal M, Frydman L, Varani G (2010) Real-time multidimensional NMR follows RNA folding with second resolution. Proc Natl Acad Sci USA 107:9192-9197

Ma H, Gruebele M (2005) Kinetics are probe-dependent during downhill folding of an engineered lambda6-85 protein. Proc Natl Acad Sci USA 102:2283-2287

Morris AM, Watzky MA, Agar JN, Finke RG (2008) Fitting neurological protein aggregation kinetic data via a 2-step, minimal "Ockham's razor" model: the Finke-Watzky mechanism of nucleation followed by autocatalytic surface growth. Biochemistry 47:2413-2427

Morris AM, Watzky MA, Finke RG (2009) Protein aggregation kinetics, mechanism, and curve-fitting: a review of the literature. Biochim Biophys Acta Protein Proteomics 1794:375-397

Nakamura HK, Sasai M, Takano M (2004) Squeezed exponential kinetics to describe a nonglassy downhill folding as observed in a lattice protein model. Proteins 55:99-106

Neal S, Nip AM, Zhang H, Wishart DS (2003) Rapid and accurate calculation of protein ${ }^{1} \mathrm{H},{ }^{13} \mathrm{C}$ and ${ }^{15} \mathrm{~N}$ chemical shifts. J Biomol NMR 26:215-240

Osváth S, Sabelko JJ, Gruebele M (2003) Tuning the heterogeneous early folding dynamics of phosphoglycerate kinase. J Mol Biol 333:187-199

Sabelko J, Ervin J, Gruebele M (1999) Observation of strange kinetics in protein folding. Proc Natl Acad Sci USA 96:6031-6036

Thusius D, Dessen P, Jallon JM (1975) Mechanisms of bovine liver glutamate dehydrogenase self-association. I. Kinetic evidence for a random association of polymer chains. J Mol Biol 92:413-432

Van Nuland NAJ, Forge V, Balbach J, Dobson CM (1998) Real-time NMR studies of protein folding. Acc Chem Res 31:773-780

Watzky MA, Finke RG (1997) Transition metal nanocluster formation kinetic and mechanistic studies. A new mechanism when hydrogen is the reductant: slow, continuous nucleation and fast autocatalytic surface growth. J Am Chem Soc 119:10382-10400

Wegner A, Engel J (1975) Kinetics of the cooperative association of actin to actin filaments. Biophys Chem 3:215-225

Wetzel R (2006) Kinetics and thermodynamics of amyloid fibril assembly. Acc Chem Res 39:671-679

Zeeb M, Balbach J (2004) Protein folding studied by real-time NMR spectroscopy. Methods 34:65-74 